\title{
Development of SyNRAC
}

\author{
Hitoshi Yanami $^{1,2}$ and Hirokazu Anai ${ }^{1,2}$ \\ 1 Information Technology Core Laboratories, Fujitsu Laboratories Ltd, \\ Kamikodanaka 4-1-1, Nakahara-ku, Kawasaki 211-8588, Japan \\ yanami@flab.fujitsu.co.jp, anai@jp.fujitsu.com \\ 2 CREST, Japan Science and Technology Agency, \\ Kawaguchi Center Building, 4-1-8, Honcho, Kawaguchi 332-0012, Japan
}

\begin{abstract}
We present newly implemented procedures in SyNRAC, which is a Maple package for solving real algebraic constraints derived from various engineering problems. The current version of SyNRAC has added quantifier elimination (QE) by cylindrical algebraic decomposition (CAD) as well as QE by virtual substitution for weakly parametric linear formulas. We also show an application of CAD-based QE to the common Lyapunov function problem.
\end{abstract}

\section{Introduction}

Recently symbolic computation methods have been gradually applied to solving engineering problems, which has been caused by the efficient symbolic algorithms introduced and improved for these few decades and by the advancement of computer technology that has hugely increased the CPU power and memory capacity.

We have been developing a Maple toolbox, called SyNRAC, for solving real algebraic constraints. SyNRAC stands for a Symbolic-Numeric toolbox for Real Algebraic Constraints and is aimed at being a comprehensive toolbox including a collection of symbolic, numerical, and symbolic-numeric solvers for real algebraic constraints derived from various engineering problems. When we say a real algebraic constraint, what we have in mind is a first-order formula over the reals. Our main method is quantifier elimination (QE), which removes the quantified variables in a given formula to return a quantifier-free equivalent.

In this paper we present the newly implemented procedures in SyNRAC. In (1) two types of special QE methods as well as some simplification procedures of quantifier-free formulas had been implemented in SyNRAC. Besides, the current version of SyNRAC provides the following:

- general QE by cylindrical algebraic decomposition (CAD)

- special QE by virtual substitution for weakly parametric linear formulas.

CAD-based QE is called general in the sense that it can deal with any type of formula, without thought of the efficiency. Historically, the CAD-based approach 
preceded the special QE methods we had already implemented in SyNRAC. We implemented special QE first because there was a good class of formulas to which many practical problems could be reduced and a much more efficient special QE method was applicable [2, 3.

The latter procedure is an improved special QE procedure based on 4 for a subclass of the linear formulas - the weakly parametric linear formulas. We have implemented more efficient QE specialized for the class, which can reduce the size of an elimination set roughly by a factor of two at each stage.

This paper is organized as follows. We briefly describe CAD and show the commands on CAD in SyNRAC in Section 2. In Section 3, we present special QE by virtual substitution for weakly parametric linear formulas and compare the procedure in SyNRAC with a previous one. In Section 4, we show an example problem to which SyNRAC's CAD command can apply. We end with a conclusion in Section 5.

\section{Cylindrical Algebraic Decomposition}

Cylindrical algebraic decomposition (CAD) was discovered by Collins in 1973; see [5] for his monumental work. Collins also proposed a general QE algorithm based on CAD, which provided a powerful method for solving real algebraic constraints.

Let $A$ be a finite subset of $\mathbb{Z}\left[x_{1}, \ldots, x_{n}\right]$. An algebraic decomposition for $A$ is a collection of mutually disjoint, semi-algebraic, $A$-invariant sets that partitions the Euclidean $n$-space $E^{n}$. To define the term cylindrical, we explain three parts of a CAD procedure - the projection phase, the base phase, and the lifting phase.

In the projection phase of a CAD, the PROJ function plays a central role. Let $r$ be an integer greater than 1. PROJ maps a finite set of integral polynomials in $r$ variables to a finite set of integral polynomials in $r-1$ variables: for $A_{r} \subset$ $\mathbb{Z}\left[x_{1}, \ldots, x_{r}\right], \operatorname{PROJ}\left(A_{r}\right) \subset \mathbb{Z}\left[x_{1}, \ldots, x_{r-1}\right]$. For a given $A \subset \mathbb{Z}\left[x_{1}, \ldots, x_{n}\right]$, we obtain a list

$$
A=A_{0} \stackrel{\text { PROJ }}{\longmapsto} A_{1} \stackrel{\text { PROJ }}{\longmapsto} A_{2} \stackrel{\text { PROJ }}{\longmapsto} \cdots \stackrel{\text { PROJ }}{\longmapsto} A_{n-1},
$$

where $A_{i} \subset \mathbb{Z}\left[x_{1}, \ldots, x_{n-i}\right]$.

In the base phase we partition $E^{1}$ by using a set of univariate polynomials $A_{n-1} \subset \mathbb{Z}\left[x_{1}\right]$; we find all the real zeros of $A_{n-1}$ and partition $E^{1}$ into $A_{n-1}$-invariant regions that consist of the zeros of $A_{n-1}$ and the remaining open intervals. These points and intervals are called sections and sectors, respectively.

The lifting phase inductively constructs a decomposition of $E^{i+1}$ from the decomposition of $E^{i}, i=1,2, \ldots, n-1$. Suppose $D$ is a decomposition of $E^{i}$. A lifting of $D$ is a decomposition $\bar{D}$ of $E^{i+1}$ obtained by decomposing the space $R \times E^{1}$ by using $A_{n-i-1}$ for each region $R \in D$ and putting all of them together. Let $R$ be a region of a decomposition $D$ of $E^{i} . R \times E^{1}$ is decomposed by the following; Take a point $\left(p_{1}, \ldots, p_{i}\right)$ in $R$ and substitute it for $\left(x_{1}, \ldots, x_{i}\right)$ in each polynomial in $A_{n-i-1}$ to obtain a set of univariate polynomials in $x_{i+1}$; Partition $E^{1}$ into, say, $L_{0}, L_{1}, \ldots, L_{2 k+1}$ by using the roots of the polynomials 
in $x_{i+1}$; Regard $R \times L_{0}, R \times L_{1}, \ldots, R \times L_{2 k+1}$ as the resulting decomposition. The condition for this process to work is that $A_{n-i-1}$ is delineable on $R$, in other words, every pair of polynomials in $A_{n-i-1}$ has no intersections on $R$. In such a case the decomposition is independent of the choice of a sample point. A decomposition $D$ of $E^{r}$ is cylindrical if it is constructed by iterating the above lifting method, i.e., $r=1$ and $E^{1}$ is decomposed as in the base phase, or $r>1$ and $D$ is a lifting of some cylindrical decomposition $D^{\prime}$ of $E^{r-1}$.

Given a formula $\varphi$ one can construct a CAD for the polynomials of the atomic formulas in $\varphi$. The point for CAD-based $\mathrm{QE}$ is that the truth/falsehood of $\varphi$ is determined regionwise because each region in the CAD is $A$-invariant. See [5] for details.

It is the PROJ function that is crucial in a CAD procedure. The fewer polynomials PROJ produces, the more efficient the CAD program becomes. But PROJ must be constructed to maintain the delineability and make the lifting phase possible. Some improvements in the projection phase of CAD are found in $6,7,8$.

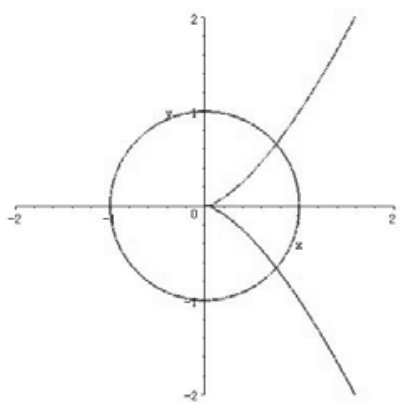

Fig. 1. The graph of A

Here we show some examples of CAD commands in SyNRAC. We construct a CAD for $A:=\left\{x^{2}+y^{2}-1, x^{3}-y^{2}\right\} \subset \mathbb{Z}[x, y]$. The graph of the two polynomials in $A$ is shown in Fig. 1. The Projection command repeats PROJ and returns $\mathrm{P}[1]=\operatorname{PROJ}^{0}(A)=A$ and $\mathrm{P}[2]=\operatorname{PROJ}(A)$.

$>$ read "synrac";

$>A:=\left[x^{\wedge} 2+y^{\wedge} 2-1, x^{\wedge} 3-y^{\wedge} 2\right]:$

$>\mathrm{P}:=\operatorname{Projection}(\mathrm{A},[\mathrm{y}, \mathrm{x}])$ :

$>\mathrm{P}[1] ; \mathrm{P}[2]$;

$$
\begin{aligned}
& {\left[x^{\wedge} 2+y^{\wedge} 2-1, x^{\wedge} 3-y^{\wedge} 2\right]} \\
& {\left[x^{\wedge} 2-1, x^{\wedge} 2-1+x^{\wedge} 3, x\right]}
\end{aligned}
$$

Next the Base command partitions $E^{1}$ by using $\mathrm{P}$ [2] and returns a list of points that represent respective sections or sectors. A rational point is taken as a sample point for a sector, and a vanishing polynomial and an isolated interval are taken for a section. There are four real roots (sections) in P [2] and they make five open intervals (sectors). 
$>\operatorname{Base}(\mathrm{P}[2], \mathrm{x})$;

$[-2,[x+1,[-1,-1]],-1 / 2,[x,[0,0]], 3 / 8$, $\left.\left[x^{\wedge} 2-1+x^{\wedge} 3,[3 / 4,7 / 8]\right], 15 / 16,[x-1,[1,1]], 2\right]$

Lastly the Lifting command makes a stack for each section or sector. Out of nine regions, we have the third and the fifth ones displayed. The third region is a sector with a rational sample point [-1/2] and the stack on it is represented in a list of five sample points of sections/sectors. The data for the fifth sector are shown in a similar way. The data for It is similar for the fifth sector, which has [3/8] as a sample point.

$>\mathrm{L}:=\operatorname{Lifting}(\mathrm{P},[\mathrm{y}, \mathrm{x}])$ :

$>$ op (L[3]); op (L[5]);

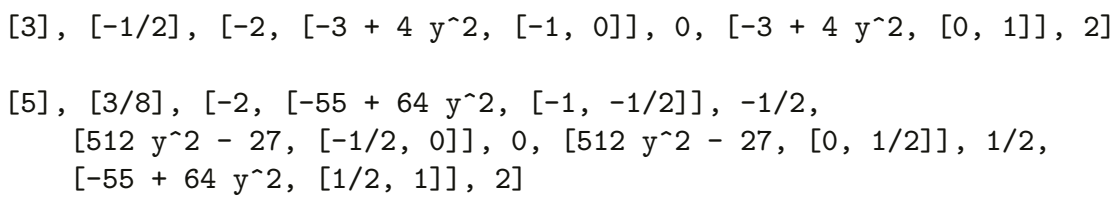

\section{Special QE for Weakly Parametric Linear Formulas}

We gave a description of a special QE algorithm by virtual substitution for linear formulas [9]. We firstly review special QE for linear formula and then explain the weakly parametric case. We will show some experimental results to compare the efficiency of the two QE procedures.

\subsection{Special QE by Virtual Substitution for Linear Formulas}

In 1988 Weispfenning [10] proposed a QE algorithm for linear formulas by virtual substitution. A formula is called linear if its atomic subformulas are all linear with respect to its quantified variables. In other words, every equality or inequality in the formula can be represented in

$$
a_{0}+a_{1} x_{1}+\cdots+a_{n} x_{n} \rho 0,
$$

where $x_{i}$ are quantified variables, $a_{i}$ are free, and $\rho$ is a relational operator, i.e., one of $\{=, \neq, \leq,<\}$.

Let $\psi\left(p_{1}, \ldots, p_{m}\right):=Q_{1} x_{1} \cdots Q_{n} x_{n} \varphi\left(p_{1}, \ldots, p_{m}, x_{1}, \ldots, x_{n}\right)$ be a linear formula, where $Q_{i} \in\{\forall, \exists\}$ and $\varphi$ is quantifier-free. By using the equivalence $\forall x \varphi(x) \Longleftrightarrow \neg(\exists x \neg \varphi(x))$, we can change the formula into its equivalent of the form $(\neg) \exists x_{1} \cdots(\neg) \exists x_{n}(\neg) \varphi$. The possible negation $(\neg)$ attached to $\varphi$ can be easily eliminated (use De Morgan's law and rewrite the atomic subformulas), which is not essential part of QE. Therefore all we are required to do is eliminate $\exists x$ in $\exists x \varphi$. In general case, all the quantifiers in the formula can be eliminated inside out by changing the innermost one to $\exists x_{i}$ and then removing it. 
To eliminate the quantified variable $\exists x$ in $\exists x \varphi$, we use a theorem in [10] saying that there exists a finite set $S$ of $x$-free terms such that

$$
\exists x \varphi \Longleftrightarrow \bigvee_{t \in S} \varphi(x / / t)
$$

holds. We note that there is a procedure transforming the expression $\varphi(x / t)$ obtained from $\varphi$ by substituting $t$ for $x$ into an equivalent formula [11. We denote the resulting formula by $\varphi(x / / t)$. Such a set $S$ is called an elimination set for $\exists x \varphi$. We obtain a quantifier-free formula equivalent to $\exists x \varphi$ by substituting all candidates in $S$ into $\varphi$ disjunctively.

This theorem indicates that smaller elimination sets would increase the efficiency of a QE procedure. Loos and Weispfenning [12] and Weispfenning [1] have presented a smaller elimination set to improve the algorithm. All of these algorithms had been implemented in SyNRAC.

\subsection{Special QE for Weakly Parametric Linear Formulas}

Weispfenning [4] has further improved the algorithm for a subclass of linear formulas. A linear formula is called weakly parametric if the coefficients of the quantified variables in every atomic formula $a_{0}+a_{1} x_{1}+\cdots+a_{n} x_{n} \rho 0$ are all constant. Therefore free variables occur only in the constant term $a_{0}$ in a weakly parametric linear formula.

In such a case, one can tell beforehand which half-interval should be used for each inequality and can exploit the information to dispense with as much as half an elimination set per quantified variable. See 4 for details.

\subsection{Comparison of the Two Procedures}

We compare a linear QE procedure previously implemented in SyNRAC and a specialized one for weakly parametric linear formulas. We have used nine example problems that were taken from the design problem of a PI controller. Problem 1 has five quantified variables. The number of variables increases one by one. So Problem 9 has 13 variables to be eliminated. We show Problems 1 and 2 here, leaving the others out because of a lack of space.

Problem1

$\& \operatorname{Ex}\left(\left[k_{1}, k_{2}, c_{0}, c_{1}, c_{2}\right], \quad \& a n d\left(\left[0 \leq c_{2}+\left(1 / 4 p_{1}-1\right) p_{2}{ }^{3}, 0 \leq-c_{2}+\left(1 / 4 p_{1}+1\right) p_{2}{ }^{3}\right.\right.\right.$,

$0 \leq c_{1}+\left(1 / 2 p_{1}-4\right) p_{2}{ }^{2}, 0 \leq-c_{1}+\left(1 / 2 p_{1}+4\right) p_{2}{ }^{2}, 0 \leq \bar{k}_{1}+c_{0}+\left(p_{1}-6\right) p_{2}$,

$0 \leq-k_{1}-c_{0}+\left(p_{1}+6\right) p_{2}, 0 \leq k_{2}+2 p_{1}-4,0 \leq-k_{2}+2 p_{1}+4,-1 \leq c_{0}, c_{0} \leq 1$,

$\left.\left.\left.1 \leq c_{1}, c_{1} \leq 3 / 2,1 / 2 \leq c_{2}, c_{2} \leq 3 / 2\right]\right)\right)$

Problem2

$\& \operatorname{Ex}\left(\left[k_{1}, k_{2}, c_{0}, c_{1}, c_{2}, c_{3}\right], \quad \& a n d\left(\left[0 \leq c_{3}+\left(1 / 4 p_{1}-1\right) p_{2}{ }^{4}, 0 \leq-c_{3}+\left(1 / 4 p_{1}+1\right) p_{2}{ }^{4}\right.\right.\right.$,

$0 \leq c_{2}+\left(1 / 2 p_{1}-6\right) p_{2}{ }^{3}, 0 \leq-c_{2}+\left(1 / 2 p_{1}+6\right) p_{2}{ }^{3}, 0 \leq c_{1}+\left(p_{1}-15\right) p_{2}{ }^{2}$,

$0 \leq-c_{1}+\left(p_{1}+15\right) p_{2}{ }^{2}, 0 \leq k_{1}+c_{0}+\left(2 p_{1}-18\right) p_{2}, 0 \leq-k_{1}-c_{0}+\left(2 p_{1}+18\right) p_{2}$,

$0 \leq k_{2}+4 p_{1}-10,0 \leq-k_{2}+4 p_{1}+10,-1 \leq c_{0}, c_{0} \leq 1,1 \leq c_{1}, c_{1} \leq 3 / 2$,

$\left.\left.\left.1 / 2 \leq c_{2}, c_{2} \leq 3 / 2,1 / 2 \leq c_{3}, c_{3} \leq 3 / 2\right]\right)\right)$ 
Table 1. Linear QE and weakly parametric linear QE

\begin{tabular}{c|rr|rr}
\hline Problem & \multicolumn{2}{|c|}{ Linear (general) } & \multicolumn{2}{|c}{ Weakly parametric linear } \\
\hline & time (sec) \# atoms & time (sec) & \# atoms \\
\hline 1 & 10.406 & 112 & 0.093 & 36 \\
2 & 2.708 & 608 & 0.156 & 92 \\
3 & 12.281 & 3072 & 0.320 & 232 \\
4 & 513.406 & 69632 & 0.626 & 560 \\
5 & - & - & 1.360 & 1312 \\
6 & - & - & 2.968 & 3008 \\
7 & - & - & 6.733 & 6784 \\
8 & - & - & 16.375 & 14848 \\
9 & - & - & 39.828 & 32768 \\
\hline On a Pentium IV 2.4 GHz processor with 1 GB memory
\end{tabular}

The results are shown in Table 1. The previous procedure could not solve Problem 5 and beyond in an hour, while the algorithm specialized for weakly parametric formula solved all of the example problems. Let us compare the computing time per elimination. At Problem 1 the procedure for weakly parametric formulas has computed $(0.406 / 0.093)^{1 / 5} \approx 1.34$ times faster than the previous one. The corresponding ratios grow gradually and reach 2.31 at Problem 4 . We suppose that simplification procedures have a more effect on the computing time when a formula gets larger.

\section{Application of CAD-Based QE}

In this section we show an application of CAD to a practical problem. The common Lyapunov function problem is a problem that studies the existence of a common Lyapunov function for a set of linear time-invariant systems. The problem often arises in stability analysis and control design of various types of control systems such as uncertain systems, fuzzy systems, switched systems, etc. We focus on one of the main types of common Lyapunov functions, a common quadratic Lyapunov function (CQLF). See [13, 14, 15, for details.

One important issue of a CQLF problem is to find an existence condition of CQLF. For a given set of stable constant linear systems, we can verify whether the systems share a CQLF and construct the CQLF if they do with some numerical semidefinite programming (SDP) package. We consider here the problem of finding symbolic existence conditions on system matrices such that these systems share a common Lyapunov function. An existence condition provides us stability regions of parameters for control systems. Although there are some attempts to resolve this so far, only partial results are obtained. The CQLF problems to compute an existence condition can be solved by using QE systematically; see [16. 
Common Lyapunov Function Problem: We consider a set of continuoustime linear time-invariant systems

$$
\dot{\mathbf{x}}=\mathbf{A}_{c i} \mathbf{x}, \mathbf{x} \in \mathbb{R}^{n}, \mathbf{A}_{c i} \in \mathbb{R}^{n \times n}, i=1, \ldots, q .
$$

The set of systems (1) is said to have a CQLF if there exists a symmetrical positive definite matrix $\mathbf{P}=\mathbf{P}^{T}>0$ such that the following Lyapunov inequalities

$$
\mathbf{P A}_{c i}+\mathbf{A}_{c i}^{T} \mathbf{P}<0, \forall i=1, \ldots, q
$$

are satisfied. Then the CQLF is $\mathbf{V}(\mathbf{x})=\mathbf{x}^{T} \mathbf{P} \mathbf{x}$.

Solving Common Lyapunov Function Problem by QE: We consider two Hurwitz stable continuous-time linear time-invariant systems, i.e., the case $n=2$ and $q=2$ in (11):

$$
\dot{\mathbf{x}}=\mathbf{A}_{c i} \mathbf{x}, \mathbf{x} \in \mathbb{R}^{2}, \mathbf{A}_{c i} \in \mathbb{R}^{2 \times 2}, i=1,2 .
$$

Moreover let $\mathbf{A}_{c 1}, \mathbf{A}_{c 2}$ be

$$
\mathbf{A}_{c 1}=\left[\begin{array}{cc}
x & 0 \\
y & -1
\end{array}\right], \mathbf{A}_{c 2}=\left[\begin{array}{cc}
0 & 1 \\
-1 & -2
\end{array}\right],
$$

respectively, where $x, y \in \mathbb{R}$ are system parameters. We have the following theorem.

Theorem 1. (R.N.Shorten et.al. [14]) A necessary and sufficient condition for the two second-order systems (3) to have a CQLF is

$$
\begin{aligned}
& R e\left(\lambda\left(c o\left(\mathbf{A}_{c 1}, \mathbf{A}_{c 2}\right)\right)\right)<0, \\
& \operatorname{Re}\left(\lambda\left(\operatorname{co}\left(\mathbf{A}_{c 1}, \mathbf{A}_{c 2}^{-1}\right)\right)\right)<0,
\end{aligned}
$$

where co(.) denotes the convex hull (polytope) of matrices: $\operatorname{co}(\mathbf{X}, \mathbf{Y})=\{\alpha \mathbf{X}+$ $(1-\alpha) \mathbf{Y}: \alpha \in[0,1]\}, \lambda(\mathbf{X})$ denotes the eigenvalues of matrix $\mathbf{X}$ and $\operatorname{Re}($. denotes the real part of a complex number.

This implies that our desired condition is that all roots of characteristic polynomials of $\operatorname{co}\left(\mathbf{A}_{c 1}, \mathbf{A}_{c 2}\right)$ and $\operatorname{co}\left(\mathbf{A}_{c 1}, \mathbf{A}_{c 2}^{-1}\right)$ locate within a left half of the Gaussian plane for $\alpha \in[0,1]$. The conditions can be reduced to a set of polynomial inequalities by using the well-known Liènard-Chipart criterion. Then we can apply QE for the polynomial inequalities.

Now we compute feasible regions of $x, y$ so that the systems (3) have a CQLF by QE. As mentioned above, by applying the Liènard-Chipart criterion to characteristic polynomials of $\operatorname{co}\left(\mathbf{A}_{c 1}, \mathbf{A}_{c 2}\right)$ and $\operatorname{co}\left(\mathbf{A}_{c 1}, \mathbf{A}_{c 2}^{-1}\right)$, the CQLF existence condition can be described by the following formula:

$$
\begin{aligned}
\forall \alpha \quad((0 \leq \alpha \leq 1) \Rightarrow & \left(2-\alpha-x \alpha>0 \wedge 1+y \alpha-\alpha^{2}-x \alpha^{2}-y \alpha^{2}>0 \wedge\right. \\
& \left.\left.1-2 \alpha-2 x \alpha-y \alpha+\alpha^{2}+x \alpha^{2}+y \alpha^{2}>0\right)\right) .
\end{aligned}
$$

Applying CAD-based QE in SyNRAC to (7D), the output turns out to be

$$
(x<0 \wedge-2-2 \sqrt{-x}<y<2 \sqrt{-x}-2 x) .
$$




\section{Conclusion}

We have presented a newly developed functions in Maple-package SyNRAC. The current version of SyNRAC provides quantifier elimination by virtual substitution up to quadratic formulas and CAD-based QE, as well as some standard simplifiers. The new features greatly extend the applicability and tractability of SyNRAC for solving real algebraic constraints in engineering. As an application of SyNRAC, we have treated a common Lyapunov function problem.

We proceed to implement other known QE algorithms and improve them, and are setting about developing symbolic-numeric algorithms. We also plan to develop a toolbox for parametric robust control design on MATLAB using SyNRAC as a core engine.

\section{References}

1. Anai, H., Yanami, H.: SyNRAC: A Maple-package for solving real algebraic constraints. In: Proceedings of International Workshop on Computer Algebra Systems and their Applications (CASA) 2003 (Saint Petersburg, Russian Federation), P.M.A. Sloot et al. (Eds.): ICCS 2003, LNCS 2657, Springer (2003) 828-837

2. Anai, H., Hara, S.: A parameter space approach for fixed-order robust controller synthesis by symbolic computation. In: Proceedings of IFAC World Congress on Automatic Control b’02. (2002)

3. Anai, H., Yanami, H., Hara, S.: SyNRAC: a maple-package for solving real algebraic constraints toward a robust parametric control toolbox. In: Proceedings of SICE Annual Conference 2003 (Fukui, Japan). (2003) 1716-1721

4. Weispfenning, V.: Simulation and optimization by quantifier elimination. Journal of Symbolic Computation 24 (1997) 189-208 Special issue on applications of quantifier elimination.

5. Collins, G.E.: Quantifier elimination for real closed fields by cylindrical algebraic decomposition. In Caviness, B., Johnson, J., eds.: Quantifier Elimination and Cylindrical Algebraic Decomposition. Texts and Monographs in Symbolic Computation. Springer, Wien, New York (1998) 85-121

6. Hong, H.: An improvement of the projection operator in cylindrical algebraic decomposition. In Caviness, B., Johnson, J., eds.: Quantifier Elimination and Cylindrical Algebraic Decomposition. Texts and Monographs in Symbolic Computation. Springer, Wien, New York (1998) 166-173

7. McCallum, S.: An improved projection operation for cylindrical algebraic decomposition. In Caviness, B., Johnson, J., eds.: Quantifier Elimination and Cylindrical Algebraic Decomposition. Texts and Monographs in Symbolic Computation. Springer, Wien, New York (1998) 242-268

8. Brown, C.W.: Improved projection for cylindrical algebraic decomposition. Journal of Symbolic Computation 32 (2001) 447-465

9. Anai, H., Yanami, H.: SyNRAC: a Maple-package for solving real algebraic constraints. In: Proceedings of International Conferences on Computational Science. Volume 2657 of LNCS., Springer (2003) 828-837

10. Weispfenning, V.: The complexity of linear problems in fields. Journal of Symbolic Computation 5 (1988) 3-27 
11. Weispfenning, V.: Quantifier elimination for real algebra-the quadratic case and beyond. Applicable Algebra in Engineering Communication and Computing 8 (1997) 85-101

12. Loos, R., Weispfenning, V.: Applying linear quantifier elimination. The Computer Journal 36 (1993) 450-462 Special issue on computational quantifier elimination.

13. Shorten, R.N., Narendra, K.S.: Necessary and sufficient conditions for the existence of a common quadratic lyapunov function for $\mathrm{m}$ stable second order linear timeinvariant systems. Proc. of ACC (2000) 359-363

14. Shorten, R.N., Narendra, K.S.: Necessary and sufficient conditions for the existence of a common quadratic lyapunov function for two stable second order linear timeinvariant systems. Proc. of ACC (1999) 1410-1414

15. Mori, Y.: Investigation on common lyapunov function: Toward complete analysis. Kyoto Institute of Technology, Doctoral Thesis, (in Japanese) (2002)

16. Nguyen, T.V.: Common lyapunov function problem: Quadratic and infinity-norm functions. Kyoto Institute of Technology, Master Thesis (2003) 